\title{
Lyman Lines in Prominences and Filaments: NLTE Modelling Strategies
}

\author{
P. Heinzel
}

Astronomical Institute, Academy of Sciences of the Czech Republic CZ-25165 Ondřejov, Czech Republic

\begin{abstract}
We briefly summarize the most important aspects of NLTE formation of hydrogen Lyman lines in prominences and filaments. A modified MALI code of Heinzel (1995) is described and its applicability to the interpretation of SOHO/SUMER data is discussed.
\end{abstract}

\section{Introduction}

A sophisticated NLTE modelling of the first few hydrogen Lyman lines (L $\alpha$ to $\mathrm{L} \delta$ ) in isothermal and isobaric slabs has been performed, taking into account the partial redistribution (PRD) scattering physics, multilevel transitions and realistic incident radiation fields. In 1D, prominences are modelled as finite vertical slabs (Gouttebroze et al. 1993) and filaments as horizontal slabs (e.g. Mein et al. 1996). For 2D models see Paletou (1995) and Gorshkov (1997). Typically a 5-level plus continuum hydrogen model atom was used. Only Gouttebroze et al. (1993) have considered up to 30 levels, but Lyman transitions higher than L $\delta$ have been put into detailed radiative balance. In this contribution we summarize the strategies of how to extend such modelling to higher or even very high members of the Lyman series.

\section{Higher Lyman Lines}

Detailed study of NLTE line formation of higher Lyman lines requires an effective treatment of a multilevel hydrogen atom with a large number of explicit transitions for which the transfer equation must be solved. For example, to compute the intensity profiles of the four Lyman lines recently detected in a filament by SUMER, i.e., L $\delta$ to L-7 (see Schmieder et al. 1998, these proceedings), we have used a 12-level hydrogen model atom with 78 transitions including the continua (note that all subordinate continua are considered to be optically thin with radiative rates fixed by the incident radiation fields). As shown by Heinzel et al. (1997), for higher Lyman lines in prominences and filaments, the Stark broadening dominates the natural one. On the other hand, the importance of PRD is substantially lowered and one can treat higher members in the complete redistribution (CRD). Such multilevel treatment also requires an adequate routine to evaluate the collisional rates (Gouttebroze - private communication) and the full set of realistic incident radiation fields. The latter one has been taken from SOHO/SUMER measurements (for details see Heinzel et al. 1997). 


\section{MALI Code for Multilevel Problems}

Current MALI (Multilevel Accelerated Lambda Iterations) techniques are capable of treating a large number of atomic levels and explicit transitions selfconsistently (Rybicki and Hummer 1991, Heinzel 1995, Paletou 1995), thus replacing the assumption of detailed radiative balance in higher Lyman lines. For very high members, the line ovelapping must be properly taken into account. For higher densities, the level-occupation-probability formalism has to be considered when dealing with Lyman lines close to the series limit (Hubený et al. 1994). We have modified our prominence/filament MALI code in order to include an arbitrary number of hydrogen levels. For all corresponding transitions we specify the incident radiation fields (boundary conditions). Stark broadening parameters for higher Lyman lines are estimated according to Gouttebroze et al. (1978). NLTE simulations proceed in two steps. First, the linearized statisticalequilibrium equations are solved for a 5-level model atom in order to determine the ionization structure (depth variations of the electron density). Next, we use the full number of levels and solve the linear MALI problem for prescribed electron density.

\section{Temperature Structure}

As demonstrated by Schmieder et al. (1998, these proceedings) and Heinzel et al. (1997), SOHO/SUMER data do indicate that the prominence-corona transition region (PCTR) must be considered when interpreting higher Lyman lines, particularly in the case of filaments where no incident radiation falls on their top. To account for a temperature gradient in PCTR, we have replaced our previous isothermal models with new ones considering the temperature variations with depth (column mass). This automatically requires that a depth-dependent PRD must be accounted for, and we thus evaluate the redistribution matrices at each particular depth. However, a realistic determination of the PCTR temperature structure is rather difficult (i.e., using trial and error), and we plan to apply an effective optimization technique to ensure the best fits to all observed lines.

\section{References}

Gorshkov, A.B. 1997, in Science with Themis, (eds.) N. Mein and S. SahalBréchot, Observatoire de Paris, p. 273

Gouttebroze et al. 1978, ApJ, 225, 655

Gouttebroze, P., Heinzel, P. and Vial, J.-C. 1993, A\&AS, 99, 513

Heinzel, P. 1995, A\&A, 299, 563

Heinzel, P., Schmieder, B. and Vial, J.-C. 1997, in The Corona and Solar Wind Near Minimum Activity, ESA-SP 404, p. 427

Hubený, I., Hummer, D.G. and Lanz, T. 1994, A\&A, 282, 151

Mein, N. et al. 1996, A\&A, 309, 275

Paletou, F. 1995, A\&A, 302, 587

Rybicki, G.B. and Hummer, D.G. 1991, A\&A, 245, 171 